\title{
LYMIPHSPIRATION
}

\section{CLEANING THE BRAIN THROUGH TURBULENT GLYMPHATIC FLOW: THE WASHING MACHINE HYPOTHESIS}

\author{
P.H. Kuo
}

Department of Medical Imaging, Medicine, and Biomedical Engineering, University of Arizona, Tucson, Arizona, USA

\section{ABSTRACT}

In a thought experiment, a "washing machine" model is proposed based on turbulent flow from complex multi-dimensional forces to characterize fluid dynamics in the brain. The glymphatic system's hypothetical role in this system is illustrated in a series of diagrams. Implications of this model are discussed in terms of normal physiology and a variety of pathologic conditions such as brain atrophy and Alzheimer disease.

Keywords: glymphatic system, "washing machine" model, turbulent flow, meningeal lymphatics, cerebrospal fluid, brain a trophy, Alzheimer disease

A critical mechanism for the evolution of big-brained, long-lived Homo sapiens is the ability to maintain homeostasis of the brain. Knowledge of the structure and function of the glymphatic system and meningeal lymphatic vessels (MLV) has dramatically increased in recent years, but there is still much to understand (1-6). In particular, what is the pattern of glymph flow in the brain? To maximize flow rate through the brain parenchyma, glymph should take the simplest, most direct route from the periarterial space straight to the perivenous space (Fig. 1); however, this simple model should only work when there is an ample supply of fluid. The tight physical constraints of the relatively large volume of brain and spinal cord compared to the small volume of their bony confinement (cranium and spinal canal) leaves little room for cerebrospinal fluid (CSF). Cleansing the brain and spinal cord of contents may therefore be analogous to cleaning laundry with only a small amount of water. So how do you get your clothes clean if you do not have much water? Adding detergent to the laundry may be a good idea for your clothes but is not a good idea for the health of your brain. If you are left with just a small volume of clean water (i.e., CSF) to clean your dirty clothes (i.e., brain), you do not do a quick rinse (i.e., simple, laminar-type glymph flow from periarterial to perivenous space as depicted in Fig. 1) and remove little dirt. Rather, you put those dirty clothes in the washing machine, let the machine agitate the water back and forth in the clothes for an extended period of time to effectively clean, and then send the dirty water down the drain (i.e., MLV).

So, we agree that the washing machine is a robust method for cleaning your clothes with a limited amount of water, but how can we extend this analogy to cleaning your brain with glymph? Let us conduct a Gedankenexperiment (thought experiment), which inherently requires extrapolating on hypotheses in this rapidly evolving, complex field with still many 




Fig 1. Model of glymphatic flow following the most direct route through brain parenchyma. Hypothetical model of glymphatic flow following the simplest, most direct route from the periarterial space, through the brain parenchyma, and into the perivenous space. Red and blue tubular structures represent arteries and veins, respectively. Dashed lines adjacent to the arteries and veins represent the astroglial endfeet with aquaporin channels that surround the blood vessels in a co-axial fashion. The periarterial and perivenous spaces are represented by space between the vasculature (red and blue tubular structures) and the astroglial endfeet (dashed lines). Within these perivascular spaces, fluid flows as depicted by the orange and blue arrows. Glymphatic fluid (green arrows) with solutes flows through the brain parenchyma via the shortest, most direct route and drains out along the perivenous space and into the meningeal lymphatic vessel (green cylinder on the right).

unknowns. The answer may lie in the forces that drive glymph flow: pulse pressure on the arterial-side and respiratory-driven pressure changes on the CSF-side (7). Changes in CSF pressures are potentially transmitted throughout the brain via the ventricles, cisterns, and subarachnoid/perivascular spaces (8). Remember that the adult skull does not expand so these changes in pressure are transmitted to the brain parenchyma and the glymphatic fluid within it, analogous to repeatedly squeezing and releasing clothes to exchange the water within the clothes. Of great potential importance is the very different frequencies of the cardiac and respiratory cycles. In addition to those different frequencies, superimpose heart rate and respiratory variability with resulting variability in amplitudes, and you have the makings of constantly changing flow patterns, the hypothetical mechanism for a deep clean of the brain. It is interesting to think that the importance of heart rate variability and sigh- ing/respiratory variability to health may be in part due to its effects on the brain. Likewise, adding variability to pacemakers and ventilators and pulsatility/variability to cardiac assist devices may affect the homeostasis of the brain through this mechanism. Even changing position when you sleep may help with ensuring all parts of the brain get washed clean $(9,10)$.

For the next part of our thought experiment, first visualize the complex, non-linear, and three-dimensional vascular and CSF network of the brain. Then visualize the vector forces from that network applied at a myriad of angles to the adjacent brain parenchyma and glymph within it (Fig. 2). The changes in pressure from systole to diastole would transmit from the arteries of the brain in a radial fashion in a multitude of directions (since vessels do not travel in a straight line and branch) and drive glymph flow through the brain parenchyma towards the perivascular 
space on the venous side (Fig. $2 A$ ). The transmission of forces from the perivascular spaces to the brain parenchyma would be lower in amplitude and frequency than from the arterial side (Fig. 2B); however, the contribution of the forces from the perivascular spaces to the "washing machine effect" may be quite profound when you consider that the forces come from the perivascular spaces of both the arteries and veins. At first glance, this may seem like a design flaw, since forces from the perivenous space would impede the exit of glymph from brain parenchyma. But, if you think about it in terms of the "washing machine," it makes sense that forces pushing glymph both forward AND back are critical. Combining all these forces makes for a necessarily complex and turbulent picture (Fig. 2C). When thinking about the potential for complex patterns of glymph flow, you have to consider all four dimensions- $x, y, z$ and time. This
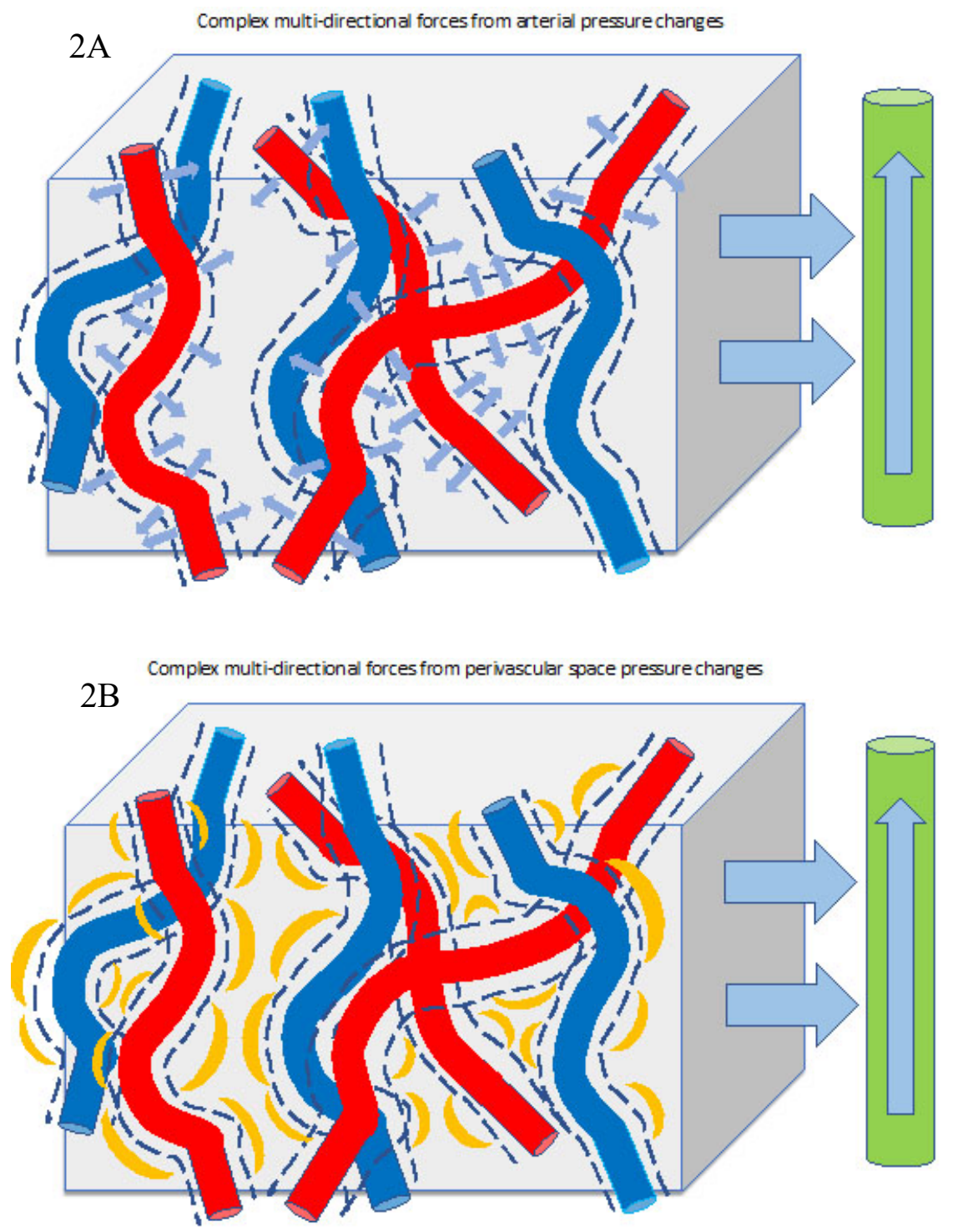


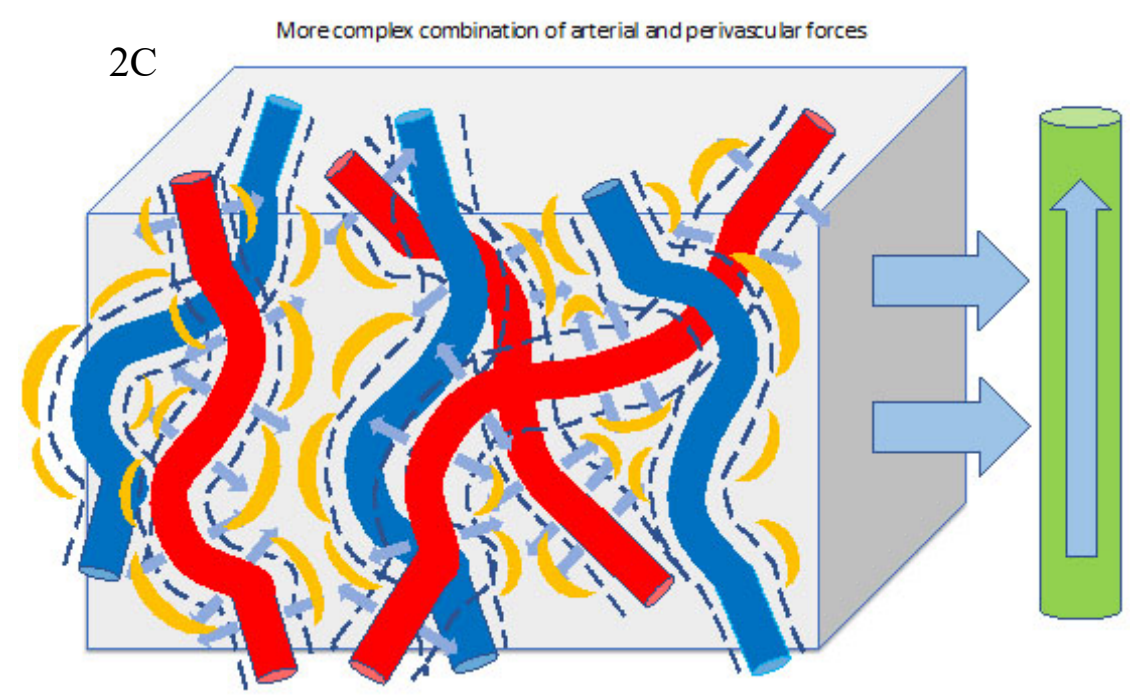

Fig. 2: Hypothetical model of forces driving glymphatic flow in the brain. A) While this model of a volume of brain is not drawn to scale, the figure is drawn to emphasize the complex 3-dimensional network of arteries, veins, and perivascular spaces. For ease of visualization, astrocytes, neurons, and extracellular matrix are not depicted. Red and blue tubular structures represent arteries and veins, respectively. Dashed lines adjacent to the arteries and veins represent the astroglial endfeet with aquaporin channels that surround the blood vessels in a co-axial fashion. The periarterial and perivenous spaces are represented by space between the vasculature (red and blue tubular structures) and the astroglial endfeet (dashed lines). Bulk flow of glymph with solutes drains out along the perivenous space and into the meningeal lymphatic vessels (green cylinder on the right). For simplicity, the arrow demonstrating flow from the perivenous space to the MLV in Fig. 1 has been replaced by the two large light blue arrows pointing left to the green cylinder representing the $M L V$. Arrows represent direction of forces from pulse pressure originating from the arteries and exerting into the adjacent periarterial space and parenchyma. The forces extend out from the artery radially (though depicted in 2-dimensions here). Branching of vessels would further add to the complexity of forces but is not shown here for simplicity and clarity. B) CSF pressure changes with respiration transmitted in ventricles, cisterns, perivascular spaces are transmitted into the fluid and tissues at a lower frequency and likely lower maximum magnitude than forces from pulse pressure. $C$ ) Combining the arterial forces depicted in (A) and the perivascular forces in (B) generate a very complex model of the forces with a multitude of directions and magnitudes and thus turbulent glymphatic flow. The very different (typically by an order of magnitude) frequencies of the forces from respiration and pulse pressure further add to the complexity and may prevent the forces of one or the other from adding together too much.

resulting chaos generates turbulent flow of glymph that moves back and forth to clean the brain with a small volume of fluid (Fig. 3).

Ultimately, the bulk direction of flow from the periarterial to perivenous side follows the flow of fluid out of the central nervous system and into the deep cervical lymphatics via the meningeal lymphatic vessels (MLV) (3-5). When modeling the system, the MLV as the drainage system may be a more important factor to ensure the net movement of glymph through the brain parenchyma, since the turbulent flow might otherwise slow bulk flow too much without an efficient outflow pathway. This general concept of maximizing "cleaning efficiency" given the highly limited volume of fluid and distances in the brain may also be built into the design of the counter-current flow of fluid in the MLV in relation to venous flow as demonstrated for the superior sagittal sinus (11).

This hypothetical "washing machine" model allows us to speculate about potential implications. As the field improves on modeling of the glymphatic/lymphatic system of the brain, supportive evidence for this model 


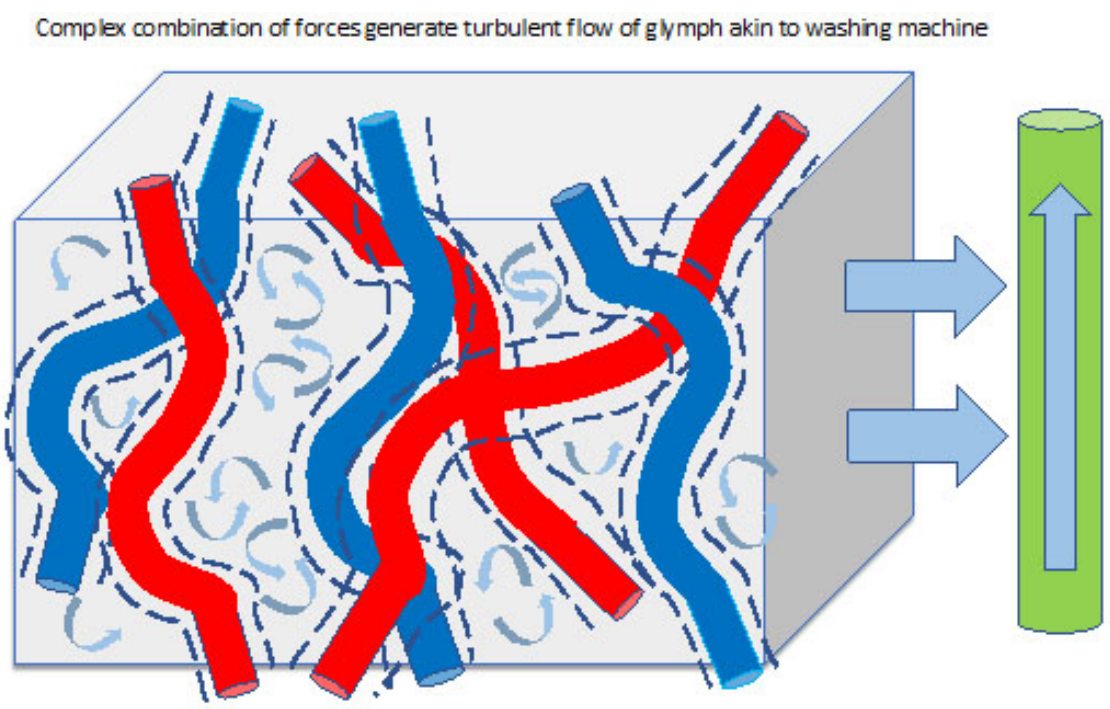

Fig. 3: Hypothetical model of the turbulent glymphatic flow in the brain necessary for optimal cleaning of the brain. The combination of complex, multi-directional forces from those structures depicted in Fig. 2 onto the adjacent brain parenchyma drive complex, turbulent flow of fluid/glymph (curved arrows) to more effectively "clean" the brain analogous to a washing machine cleaning laundry. The MLVs are the critical drainage conduits that ensure the net movement of fluid through the brain parenchyma. Working backwards, the venous system drains the cervical lymphatics, which drain the MLVS, which drain the brain parenchyma. Likewise, conditions or diseases that damage or impede flow in the $M L V$ would decrease the flow of glymph through the brain. While this three-dimensional "to and fro" motion of the fluid increases the effective cleaning volume of fluid, there must be net flow towards the meningeal lymphatics (the curved arrows do not represent circular vortices in which water and solutes remain trapped). Perhaps a loose but relatable analogy is ocean waves washing ashore since individual water molecules follow a circular orbit (up and down in the case of waves) but there is net movement of water to the shore.

would include that glymphatic volume, flow rate, and laminar flow do not adequately account for the clearing of solutes and wastes from the brain. The "washing machine" model would predict a larger effective volume of glymph. It is interesting to speculate that if volume and flow rate do increase during sleep, then the washing machine analogy may be even more accurate with the wash cycle occurring during the day and the rinse cycle at night. This potential mechanism during sleep also emphasizes the importance of treating sleep disorders such as sleep apnea and improving sleep quality and duration in the general population as well. Atrophy of the brain may result in diminished transmission of perivascular forces to the brain parenchyma and concomitantly diminished clearing of wastes from the brain. Therefore, atrophy may accelerate accumulation of wastes and pathologic proteins which may lead to further atrophy in a vicious cycle.

Exercise is known to benefit brain health and a component of this benefit may be due to its effect on heart rate, blood pressure, and breathing, which would then be the equivalent of turning the washing machine to the "heavy duty" setting. The practice of deep, regular breathing in many meditative practices may provide a voluntary mechanism for turning on the "heavy duty" wash cycle by increasing perivascular pressures to promote movement of glymph back and forth, indeed in a manner akin to the yin-yang principle. Interestingly in some Chinese health practices, the selfmassage which closes a session of meditation focuses on the scalp, face, and then neck. The MLV drain to the deep cervical nodes and 
thus increasing the lymphatic flow of the neck by massage of the head then neck could consequently result in increased flow of the MLV and thus promote flow of glymph $(12,13)$. Therefore, the practice of meditation with meditative breathing followed by head and neck massage could provide a voluntary mechanism for "heavy duty" wash followed by rinse cycle.

To support the "washing machine" hypothesis on a cellular/molecular level, one could speculate on necessary features to promote movement of fluid within the extracellular matrix (ECM) and across cellular membranes. Indeed, the brain has a unique ECM comprised primarily of non-fibrillar molecules such as proteoglycans which have a high affinity for water (14). The dynamic relationship of proteoglycans with water and pressure is better known for its critical function in articular cartilage, and perhaps that function is important in the brain as well. Extracellular beta amyloid plaques (a hallmark proteinopathy of Alzheimer's disease) may disrupt the normal flow of fluid in the extracellular matrix like overloading the washing machine and thus promote neuronal damage. The perivascular distribution of aquaporin channels may help relieve pressure from the adjacent endothelial cells by allowing the freer passage of water and thus prevent potential damage to these cells. An extrapolation from the "washing machine" hypothesis is that mislocalization of aquaporin channels as seen in a murine model of head trauma may exacerbate endothelial injury (2). The integral role of the CNS in the immune system is both increasingly understood and still mysterious, but for certain are the very different anatomies of the systemic and CNS lymphatics (15). The "washing machine" model must not prevent the effective migration of immune cells through the CNS; therefore, these immune cells may adopt a migratory phenotype that interacts with the unique ECM of the brain to respond to solute gradients or the slow flow of glymph. The "washing machine" model's effectively slower transit of fluid may increase the effective exposure time of immune cells to antigens which may compensate for lack of lymph nodes that have that role in the systemic immune system.

\section{CONCLUSION}

The "washing machine" hypothesis proposes that a combination of complex, multidirectional (and partially opposing) peri-arterial and perivenous forces drive complex, turbulent flow of glymph to more effectively "clean" the brain. This hypothesis, in conjunction with its connection to meningeal lymphatic flow, may provide important insights into maintaining health of the brain and for neuropathology.

\section{CONFLICT OF INTEREST AND DISCLOSURE}

The author is employed by Invicro. He is also a consultant, speaker, or grant recipient for Novartis, Konica Minolta, Bayer, Chimerix, Eisai, General Electric Healthcare, Blue Earth Diagnostics, and Fusion Pharma. However, these associations are unrelated to this manuscript.

\section{ACKNOWLEDGMENT}

The author would like to thank Dr. Matthew Covington for his invaluable discussions and review of the manuscript.

\section{REFERENCES}

1. Benveniste, $\mathbf{H}, \mathbf{M}$ Nedergaard: Glymphatic System. In: Neuroscience in the 21st Century. Springer, New York, NY; 2016. pp. 1945-62.

2. Plog, BA, M Nedergaard: The glymphatic system in central nervous system health and disease: past, present, and future. Annu. Rev. Pathol. 13 (2010), 379-394.

3. Louveau, A, BA Plog, S Antila, et al: Understanding the functions and relationships of the glymphatic system and meningeal lymphatics. J. Clin. Invest. 127 (2017), 3210-3219.

4. Absinta, M, SK Ha, G Nair, et al: Human and nonhuman primate meninges harbor lymphatic vessels that can be visualized noninvasively by MRI. eLife 3 (2017), 780. 
5. Benveniste, $\mathrm{H}$ : The brain's waste-removal system. Cerebrum (2018), cer-09-18.

6. Oliver, G, J Kipnis, GJ Randolph, NL Harvey: The lymphatic vasculature in the 21st century: Novel functional roles in homeostasis and disease. Cell 182 (2020), 270296. doi:10.1016/j.cell.2020.06.039.

7. Iliff, JJ, M Wang, DM Zeppenfeld, et al: Cerebral arterial pulsation drives paravascular CSF-interstitial fluid exchange in the murine brain. J. Neurosci. 33 (2013), 18190-18199.

8. Dreha-Kulaczewski, S, AA Joseph, K-D Merboldt, et al: Inspiration is the major regulator of human CSF flow. J. Neurosci. 35 (2015), 2485-2491.

9. Lee, H, L Xie, M Yu, et al: The effect of body posture on brain glymphatic transport. J. Neurosci. 35 (2015), 11034-11044.

10. Shokri-Kojori, E, G-J Wang, CE Wiers, et al: $\beta$-Amyloid accumulation in the human brain after one night of sleep deprivation. Proc. Natl. Acad. Sci. USA 115 (2018), 4483-4488.

11. Kuo, PH, C Stuehm, S Squire, K Johnson: Meningeal lymphatic vessel flow runs countercurrent to venous flow in the superior sagittal sinus of the human brain.

Tomography 4 (2018),99-104.

12. Cserr, HF, CJ Harling-Berg, PM Knopf: Drainage of brain extracellular fluid into blood and deep cervical lymph and its immunological significance. Brain Pathol. 2 (1992), 269-276.
13. Goldmann, J, E Kwidzinski, C Brandt, et al: $T$ cells traffic from brain to cervical lymph nodes via the cribroid plate and the nasal mucosa. J. Leukoc. Biol. 80 (2006), 797-801.

14. Krishnaswamy, VR, A Benbenishty, $P$ Blinder, I Sagi: Demystifying the extracellular matrix and its proteolytic remodeling in the brain: Structural and functional insights. Cell. Mol. Life Sci. 76 (2019), 3229-3248.

16. Kipnis, J. Immune system: The "seventh sense". J. Exp. Med. 215 (2018), 397-398.

Phillip H. Kuo, MD, PhD

Professor, Department of Medical Imaging University of Arizona College of Medicine 1501 N. Campbell Avenue

PO Box 245067

Tucson, AZ 85724-5067

Telephone \#: 520-626-1957

FAX \#: 520-626-1945

e-mail: pkuo@email.arizona.edu 